\title{
Designing a License Plate Recognition in Sulaimani city
}

Soran Saeed

Sulaimani Polytechnic University, Sulaimani, Iraq

Email: soran.saeed@ spu.edu.iq

\section{Abstract}

Nowadays, Automatic car license plate number recognition vitally important in our daily life because number of cars and transportation systems increases every day. It is too complicated to be fully monitored and managed by humans, for instance traffic light system, systems for tracking stolen cars, managing car parking. It is a very challenging difficulty, due to the diversity of plate formats, different scales, rotations and non-uniform illumination conditions during image acquisition. This paper mainly introduces an Automatic Number Plate Recognition System (ANPR) in Sulaimani City by using Edge Detection Techniques for plate localization and characters segmentation. Artificial Neural Network is used for character recognition.

Keywords: License plate recognition, plate edge detection and extraction, license plate character segmentation, optical character recognition, ANPR.

\section{Introduction}

The Automatic cars number plate recognition (ANPR) is a way, which is used to read the license plates on vehicles by using optical character recognition on images. They can use cameras that specifically designed for the task or other cameras. An image of cars is captured in ANPR by cameras to store the captured images as well as the text from the license plate. ANPR technology capture the front of the car then it will be processed to differentiate the plate only, which tends a specific region. The objective of the paper is to successfully locate standard Sulaimani number plate, segment characters and recognize them given a car image. The system deals with different angles, distances, scales, resolutions and illumination conditions.

\section{Background}

In Sulaimani City there is no such a system to recognize car plates. Therefore, in this research; the project is mainly focuses on finding alternative solutions to the image segmentation and character recognition problems within the License Plate Recognition. There are three main steps in this application. Firstly, it is necessary to locate and extract 
the license plate region from a larger scene image. Secondly, the car number should be extracted from the plate and the image background. Thirdly, deliver them to an OCR system for recognition. In order to identify the license plate successfully, it is necessary to locate the plate in the image captured by the camera or the recorded video. Extracting the region of plate helps in dramatically reducing both the computational expense and algorithm complexity. For instance, the image of the front of the car is larger and the plate of the car may occupy for only $10 \%$ of the image area. In addition, it is simplifying the process of segmentation and recognition, resulting in easier algorithm design and shorter computation times. The paper mainly works with the standard Sulaimani license plates but the techniques, algorithms and parameters that is be used can be adjusted easily for any similar number plates.

\section{Methodology}

In this section the process of automatic number plate recognition is addressed that consists of four main stages:

- Preprocessing the image

- License plate extraction

- Character segmentation

- Character recognition

3.1. Preprocessing the image. As previously stated, the system of automatic number plate recognition faces several difficulties. Therefore, it is essential to adjust the captured image and make it ready for the other processing steps. The first step will be conducted by applying minimum filter to the image in order to determine the dark values in the image by increasing their area. This will be conducted to make the characters and the plate edges bold, and to remove the effect of the light diagonal strips that appear in the characters and edges of the Sulaimani license plates. This is followed by the process of increasing saturation of the image to increase the separation between colors. After that, the image will be converted to grayscale [8]. Next, increasing the image contrast to separate the background from highlights [8]. 


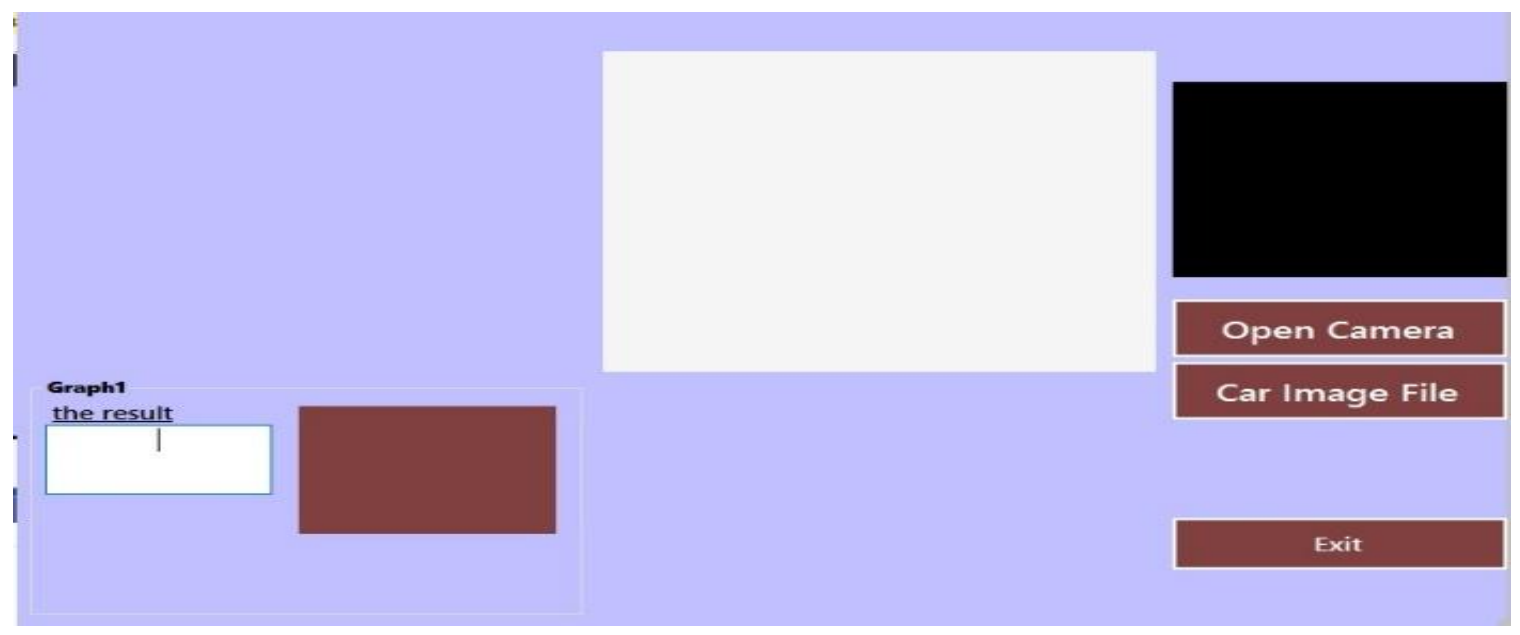

Figure 1.0: The interface of the project

3.2. License Plate extraction. In this step, the edge of the license plate is identified and the result of this step will be an image which holds the license plate only. This is conducted in two main ways.

3.2.1. Determining a large area rectangle over the license plate. In this step, a rectangle that contains the license plate is identified (this rectangle might also contain some extra parts from the four sides), and this rectangle is an input image to the next step for further processing (removing the extra parts, character segmentation then recognition) [8].

Firstly, Sobel vertical edge detection is applied to the image. Then a threshold of 36 (This value is determined using trial and error) is applied, such that every edge with magnitude less than 36 is considered false edge and is set to zero. Then a vertical projection (projecting on the $\mathrm{Y}$-axis), the edge of the determined image is taken and smoothed using an average filter with width equals nine. It is obvious that the characters of the plate along with the plate's vertical edges will have very strong vertical edges. Moreover, these edges will be determined based on the size of the object which is 20 by 40. Therefore, the approach is to cut the other parts and distinguish the plate which has the size of 20 by 40 that means the height is 20 and the width is 40 . The reason behind that is the image may contain some other objects such as the car light logos etc. That produce many vertical edges also these" false" edges may be centered in the same area so they will form a peak that may be stronger than the peak of the plate itself [8]. Now the problem is to cut the band image from the left and right to get a bounding rectangle over the license plate (Again, this rectangle doesn't have to be tight on the plate). For this purpose, a vertical Sobel edge detection is applied again, this is to decrease the effect of false edges and noise, experimentally [8]. Again, a threshold of 30 is applied 
for the same reason as before. Now, a horizontal projection of the edge detected band image is taken (projection on the X-axis) and smoothed using an average filter of large size this time, since there are gaps between the letters and the projection will have many peaks at the $\mathrm{x}$ coordinates where letters exist but it will drop down in the $\mathrm{x}$ coordinates of the gaps [8]. The bounding rectangle of the license plate is located. This is will be the input to the next step.

3.2.2. Identifying and extraction of the license plate. Using the sub-image from the last step that contains the license plate with some extra parts, the following processing is applied to this sub-image. The license plate may be skewed because of the angle of the camera while image acquisition process and it is very important to de-skew the plate to its original orientation, thus making the plate aligned with the $\mathrm{X}$ and $\mathrm{Y}$-axes. [8], [1][12]. After that, the plate has aligned with $X$ and $Y$ axes. Later, a Gaussian smoothing filter is applied to smooth the image and remove noise. Then a morphological operation will be conducted which consists of subtracting the bottom-hat of the image from the image itself is done using a structuring element of a horizontal line [7].

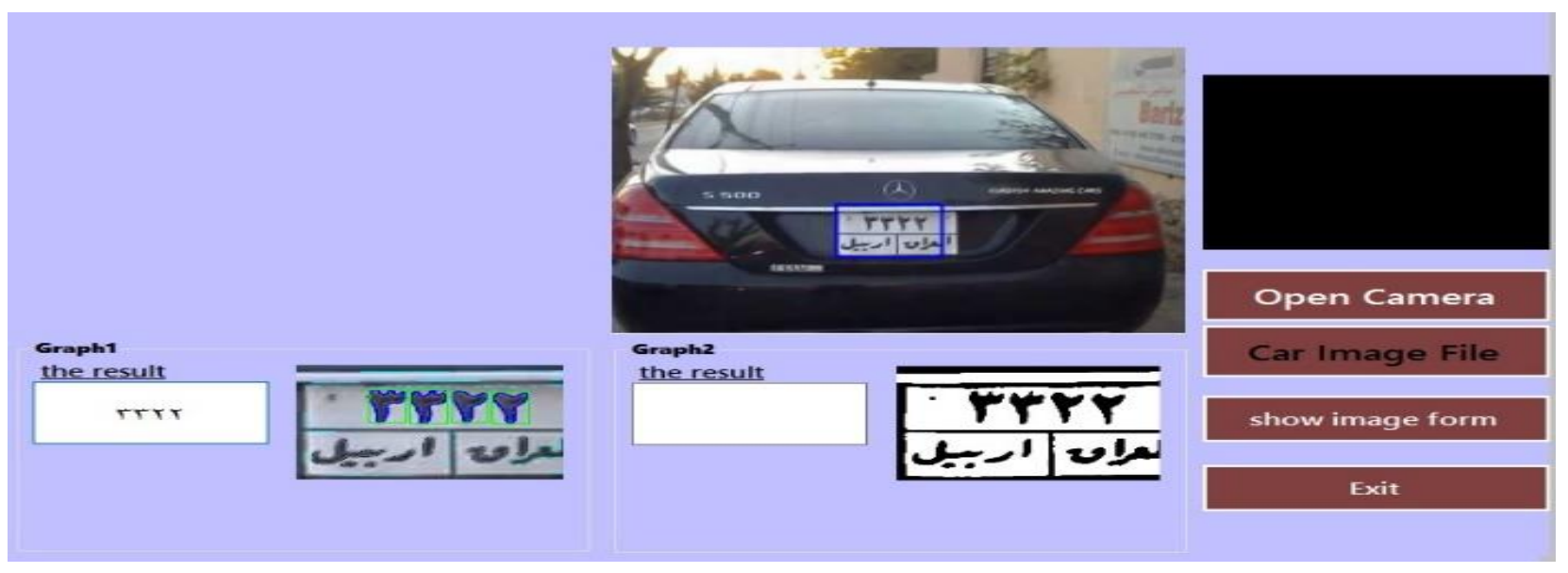

Figure2.0: Determine the plate and identifying the number

3.3. Character Segmentation. In this step, the segmentation of characters of the plate will be conducted. The result of this step is a set of monochrome images for each character in the plate. In this part, the plate image should be converted to a binary image. This is conducted using adaptive threshold. After that, noise removal process is applied. This is maintained by getting the connected components from the binary image based on the 8-neighbourhood using flood fill. Every component in this step, based on the aspect ratio of the number of pixels and component it is decided if it is a noise or not. Later, it depends on the fact that the characters of the plate have a certain range of aspect ratio and a certain range of number of pixels. Then, after the noise of the component has been removed, a maximum filter will be applied to avoid merging characters. Then the 
boundaries of the characters will be detected to distinguish each character individually. The peaks in this projection correspond to the gaps between

the characters. Therefore, if any plate number that is not fit in that range, will be rejected. In addition, a rejection measure does exist; which is equal to the variance of the characters width. After this the characters are cut according to the peaks of the previous projection and then transferred to a textbox.

3.4. Character recognition. In this step the characters are recognized and classified in the binary image which contains characters. By suing Object Character Recognition the characters will be recognized; in this step the tesseract and tessnet2 for visual studio 2015 has been used and all the characters have been practiced to be recognized better. The feature we work with in this system is the chain code of the contour of the image after dividing it into four tracks then into four sectors [10].

All the dll files imported into visual studio 2015 so the neural network is conducted inside this dll files [8][5]. The image is converted to black and white to be easily distinguish characters and extracted.

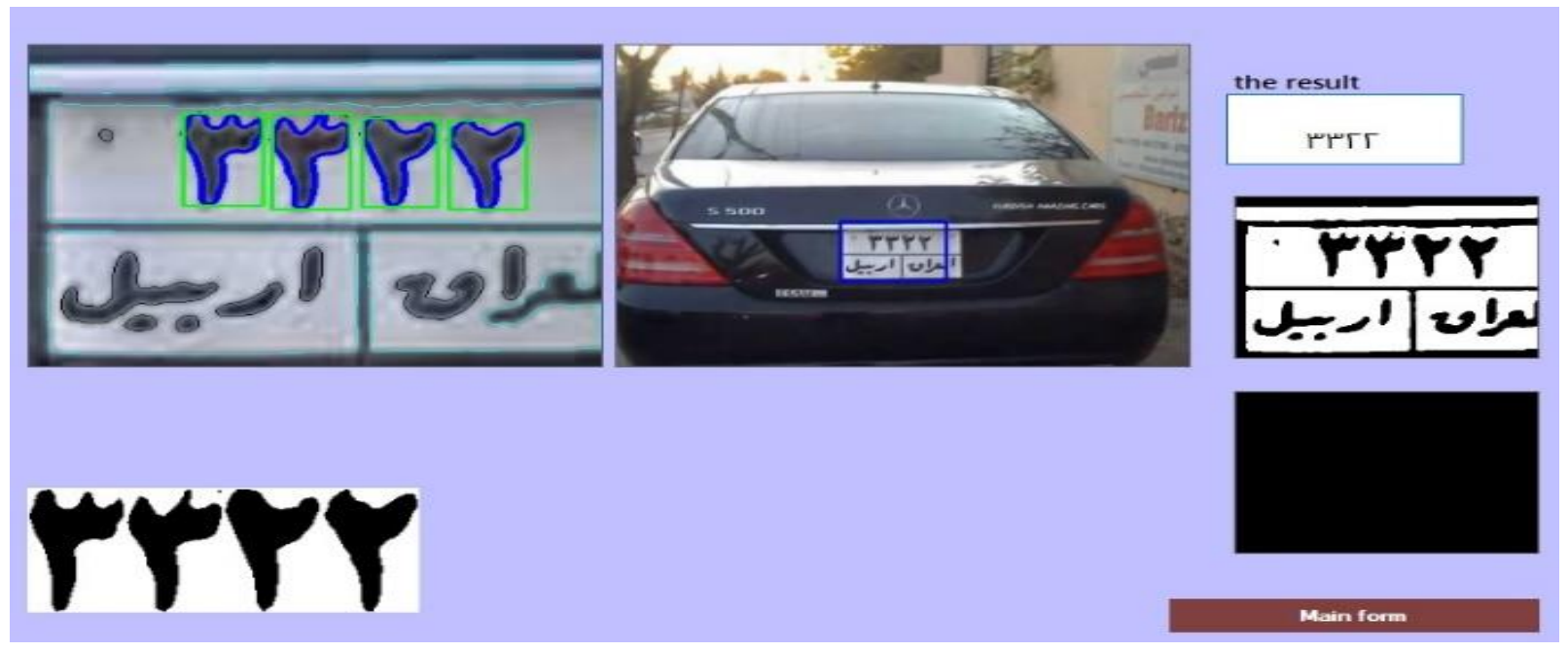

Figure3.0: Character segmentation and recognition

\section{Conclusion}

The objective of this paper was to study the plate recognition for Slulaimani, City by using, OCR and neural networks and using tesseract and tessnet 2 technologies. The process can be started either with opening image of the car or by using camera to capture the vehicle or record a video of the vehicle. The plate of the car is detected and then extracted from the image. After that, the image is converted to a binary image. Then it will be changed to black and white image this means black characters and white background. Next, the characters are extracted and transferred to a textbox. Later, it will 
be compared to the saved numbers and recognized. In the program, all the processes are conducted in the background of the program then the result can be seen in the software.

\section{Reference}

[1] B.D. Acosta, Experiments in image segmentation for automatic US license plate recognition, M.Sc. thesis, Department of Computer Science, Faculty of the Virginia Polytechnic Institute and State University (2004).

[2] C.N.E. Anagnostopoulos, I.E. Anagnostopoulos, I.D. Psoroulas, V. Loumos and E. Kayafas, License Plate Recognition From Still Images and Video Sequences: A Survey, IEEE Transactions on Intelligent Transportation Systems 9 (2008), no. 3, 377-391.

[3] C.N.E. Anagnostopoulos, I.E. Anagnostopoulos, V. Loumos and E. Kayafas, A License Plate- Recognition Algorithm for Intelligent Transportation System Applications, IEEE Transactions on Intelligent Transportation Systems 7 (2006), no. 3, 377-392.

[4] S.-L. Chang; L.-S. Chen; Y.-C. Chung; S.-W. Chen, Automatic License Plate Recognition, IEEE Transactions on Intelligent Transportation Systems 5 (2004), no. 1, $42-53$.

[5] S. Draghici, A neural network based artificial vision system for licence plate recognition, Inter-national Journal of Neural Systems 8 (1997), no. 1, 113-126.

[6] B. Enyedi, L. Konyha, C. Szombathy and K. Fazekas, Strategies for fast license plate number localization, Proceedings of the 46th International Symposium Electronics in Marine, Elmar 2004, Zadar, Croatia, June 16-18, 2004, IEEE Press (2004), 579-584 .

[7] J.-W. Hsieh, S.-H. Yu and S.-H. Yu, Morphology-based License Plate Detection from Complex Scenes, IEEE Proceedings of the 16th International Conference on Pattern Recognition, Qubec City, Canada, August 11-15, 2002, Vol. 3 (2002), 176-179.

[8] O. Martinsky, Algorithmic And Mathematical Principles Of Automatic Number Plate Recognition Systems, B.Sc. thesis, Department of Intelligent Systems, Faculty of Information Technology, Brno University of Technology (2007).

[9] J.R. Parker and P. Federl, An Approach To Licence Plate Recognition, University of Calgary 1996.

[10] H. Sarukhanyan, S. Alaverdyan, and G. Petrosyan, Automatic Number Plate Recognition System, Proceedings of the 7th International Conference on Computer Science and Information Technologies, Yerevan, Armenia, September 28-2 October, 2009, Electronic Copy of the CSIT 2009 Proceedings, 347-350. 
[11] V. Shapiro, D. Dimov, S. Bonchev, V. Velichkov and G. Gluhchev, Adaptive License Plate Image Extraction, Proceedings of the 5th international conference on Computer systems and technologies, Rousse, Bulgaria, June 17-18, 2004 (K. Boyanov), ACM New York, NY, USA (2004).

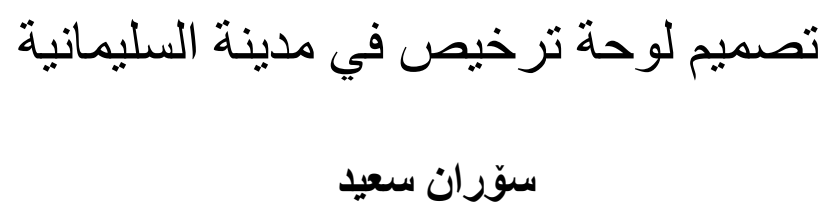

الخلاصة

في الوقت الحاضر، تمييز لوحة السيارة بشكل تلقائي أهمية حيوية في حياتنا اليومية لأن عدد السيارات وأنظمة النقل يزيد كل يوم. ومن المعقد جدا أن يتم رصدها و إدارتها بشكل كامل من قبل البشر، على سبيل المثال نظام إشار ات

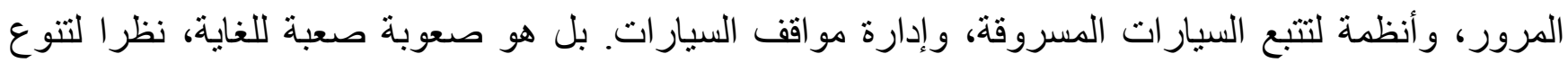

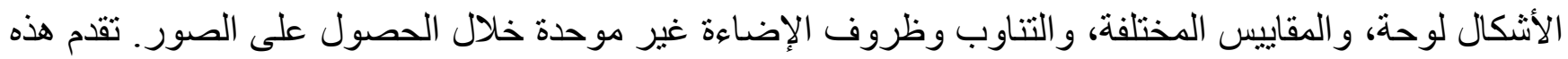
الورقة أساسا نظام التعرف التلقائي على لوحة الأرقام (ANPR) في مدينة السليمانية باستخدام تقنيات الكثف عن

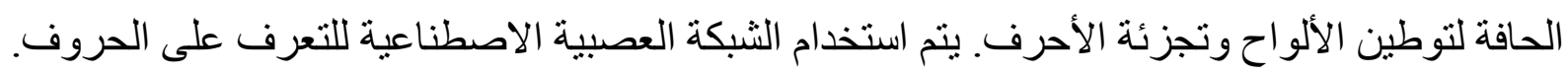
الكلمات ألمفتاحية: الاعتراف لوحة ترخيص، لوحة حافة الكثف والاستخر اج، لوحة ترخيص تجزئة حرف، التعرف الضوئي على الحروف، النع. 